\title{
Might prepatterned acetylcholine-receptor clusters on surface myotubes be a sign of neuromuscular-junction maturation failure?
}

\author{
Anna Fidziańska ${ }^{1}$, Maria Jędrzejowska ${ }^{1}$, Agnieszka Madej-Pilarczyk ${ }^{1}$, Jacek Bojakowski ${ }^{2}$ \\ ${ }^{1}$ Neuromuscular Unit, Mossakowski Medical Research Center, Polish Academy of Science, Warsaw, ${ }^{2}$ Department of Neurology, \\ Medical University of Warsaw, Poland
}

\begin{abstract}
Introduction: During human myogenesis and synaptogenesis, the first contact between multiaxonal nerve terminals and the primary myotube occurs at an early stage of gestation, then monoaxonal nerve terminals form and postsynaptic clusters of acetylcholine-receptor are modified and redistributed to the site of muscle-nerve contact.

The aim of this study is to present the ultrastructural features of muscle and motor-junction immaturity severe enough to lead to death in the first months of life.

Material and methods: Ultrastructural-level analysis was carried out on the quadriceps femoris muscle of an infant born at full term with severe respiratory distress but with normal SMN1 and IGHMBP2 genes.

Results: Arrested muscle maturation was manifested in the presence of primary and mature myotubes, prepatterned acetylcholine-receptor clusters devoid of terminal axons, lack of synapses and multiaxonal unmyelinated intramuscular nerves.

Conclusion: The "naked" prepatterned clusters observed on the surface of myotubes normally never observed in neonates might be a sign of a new genetic defect in innervation.
\end{abstract}

Key words: prepatterned cluster, primary myotube, unmyelinated multiaxonal nerves, muscle immaturity.

\section{Introduction}

Classic views on synaptogenesis have held that acetylcholine-receptor (AChR) clustering and endplate formation are induced by motor-axon contact with a myotube, by way of signals that originate from post-translation responses in the muscle. Classic studies implicated motor neuron-derived Agrin as a neural signal by which postsynaptic differentiation is induced $[1,8,17]$. These data are seen to cor- relate with ultrastructural studies of human myogenesis and synaptogenesis $[2,4,6]$. In the human foetal muscle, the first contact between multiaxonal nerve terminals and the primary myotube occurs 8-9 weeks into gestation $[5,15]$. Within the next few weeks of muscle maturation, multiaxonal nerve terminals are modified, and monoaxonal forms and postsynaptic clusters of AChR are modified and redistributed to the site of muscle-nerve contact $[5,6]$. 
Subsequent studies investigating the role of Agrin and muscle-specific tyrosine kinase (MUSK) in aneural synaptogenesis demonstrate that MuSK, but not Agrin, is required for AChR cluster formation $[13,14,18]$. Further studies revealed that positive AChR clusters are located in the central region of the muscle cell prior to the arrival of axons. This finding, termed a "prepatterned cluster", has been observed in aneural myotube cultures, as well as in mice lacking motor innervation [18]. Prepatterned AChR expression is shown to be established prior to innervation, and it is suggested that motor growth cones grow toward the prepatterned cluster $[12,16]$. All these data indicate that muscle cells play an important role in the process of synapse formation.

In our study we had an opportunity to analyze the major defect in synaptogenesis and muscle maturation in a severely affected neonate.

\section{Material and methods}

We carried out ultrastructural analysis of quadriceps femoris muscle obtained from a male infant, the first child of healthy parents born at full term with generalized hypotonia and severe respiratory distress. He had died three months after birth. Informed consent for genetic testing was obtained from the child's parents, and genomic DNA was thus extracted from its peripheral blood lymphocytes. To exclude SMN1 gene mutation, an analysis of SMN1 copy number was performed using the real-time quantitative PCR technique, as described elsewhere [9]. The SMN1 gene-dosage analysis was performed to preclude biallelic loss of the SMN1 gene, as well as compound heterozygosity of a small mutation in one allele coexisting with a deletion in the second. All 15 exons of the IGHMBP2 gene and the exon-intron boundaries were amplified using the polymerase chain reaction (PCR), and sequenced using a Big Dye Terminator Sequencing Ready Reaction kit (Applied Biosystems), before being analyzed on an ABI PRISM 373 fluorescent DNA sequencer (Applied Biosyste$\mathrm{ms})$. The primer sequences have been described previously, and so have details of the PCR protocols [7]. Specimens were fixed in buffered glutaraldehyde and embedded for electron microscopy. Microscopy techniques applied in examination are also as described previously [4].

\section{Results}

Analysis of SMN1 copy number detected 3 copies, thereby eliminating suspicions of the presence of spinal muscular atrophy (SMA) related to SMN1 gene mutations. Direct sequencing of the coding region of IGHMBP2 also failed to reveal mutations, this allowing for the exclusion, with a high degree of probability, of the diagnosis of spinal muscular atrophy with respiratory distress type 1 (SMARD1). The analyzed quadriceps femoris muscle showed marked variation in the muscle-cell diameter, with a large number of primary myotubes (Fig. 1). The latter had large, round or oval, centrally-located nuclei and myofibrils of differing density. More mature primary myotubes showed peripherally-located nuclei and cytoplasm with densely-packed myofibrils. A somewhat advanced stage of maturation was manifested by the presence of mature myotubes. In cross-section, such cells manifest a very specific pattern, appearing as two or three cells in different stages of development in close contact with each other and enveloped by a common lamina basalis (Figs. 2A-C). Among these cells arrested in maturation, it was possible to observe muscle fibers of normal diameter for the age, with well-preserved architecture. A surprising phenomenon seen in the investigated myotubes was the presence of junctional folds with a palisade-like arrangement that decorated nearly half of the myotube surface (Figs. 3A-B, 4A). Such junctional folds were short and unbranched, sometimes long and penetrating into the myotube interior, and lined with basal lamina (Fig. 4A). In some myotubes, it was possible to observe small, shallow depressions of cell membrane, which were invaginated into deep folds and devoid of terminal axons. The latter were not visible in the analyzed muscles, but a very intriguing finding was made in the vicinity of myotubes. Mononucleated cells that were poor in cytoplasm and enveloped by lamina basalis showed a tendency to arrange along the junctional palisade (Figs. 3A-B), and be separated from it by a very narrow empty space (Fig. 3A). These cells resembled abnormal Schwann cells devoid of terminal axons. Intramuscular nerves found in the investigated material exhibited profound immaturity. The unmyelinated small axons of variable diameter arranged in bundles within one Schwann cell (Fig. 4B) that formed the intramuscular nerves seen in our infant have never been observed in healthy neonates [3]. 

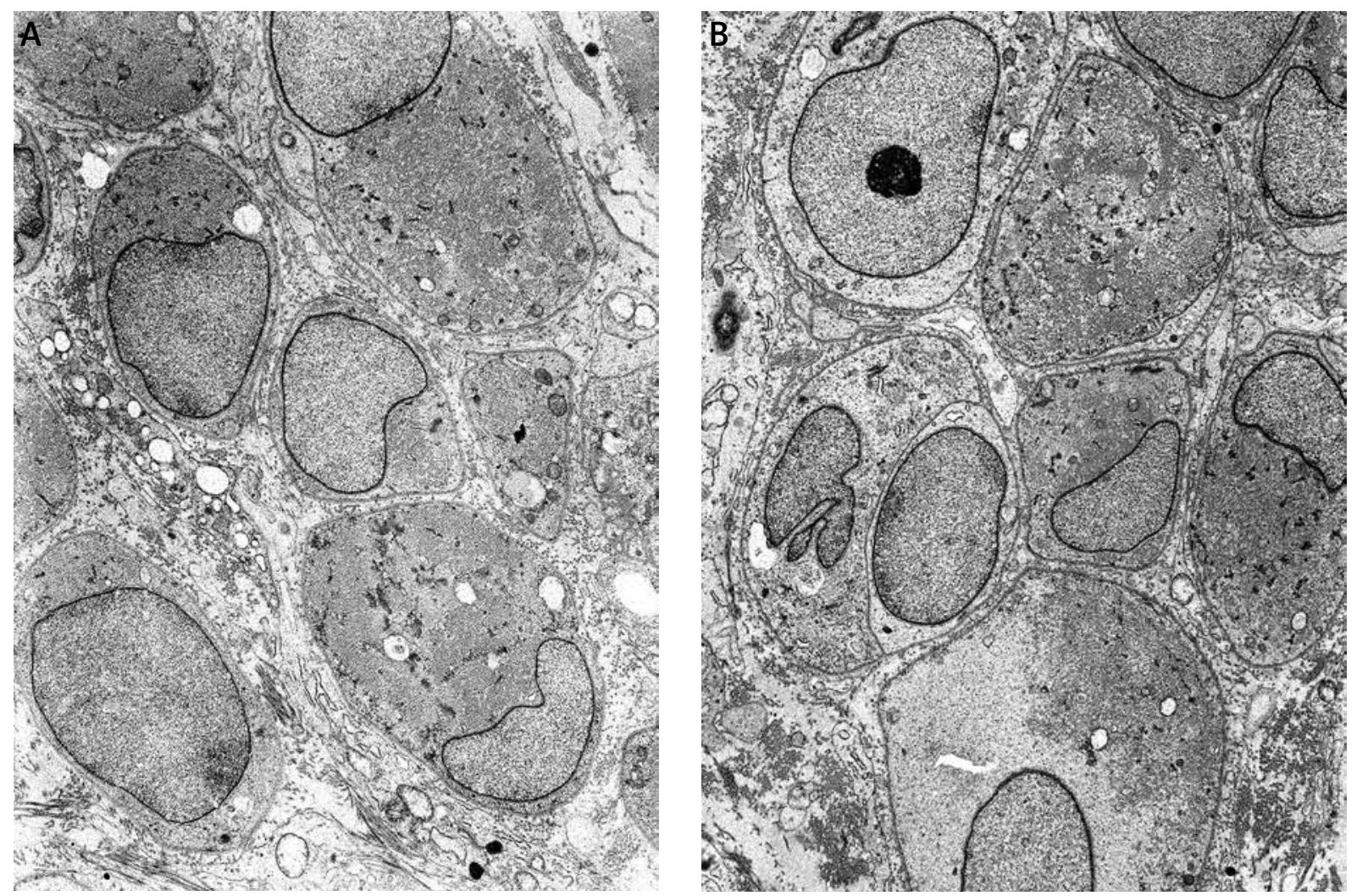

Fig. 1. Immature muscle cell with foetal features; $\times 9000$.
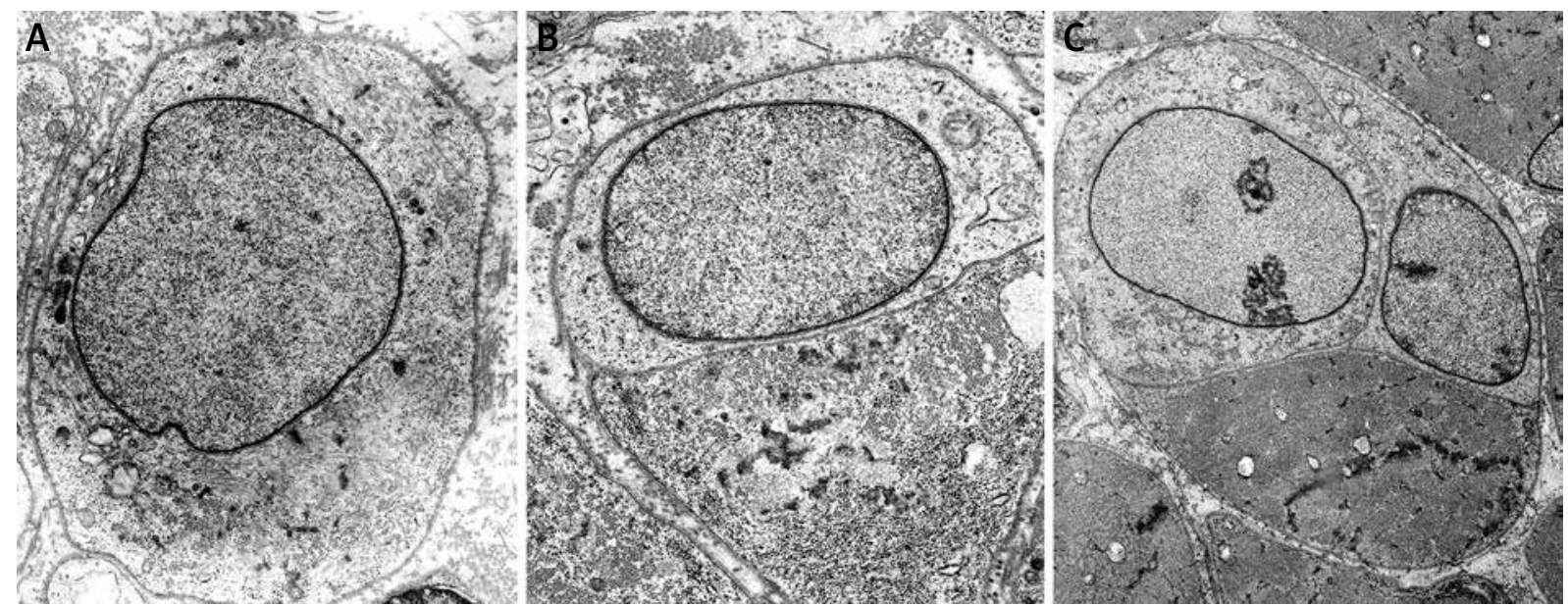

Fig. 2. Stages of mature myotube formation: A, B) primary myotube, C) mature myotube; $\times 21000$.

\section{Discussion}

Our ultrastructural study indicates the presence of profound immaturity of muscle tissue and muscle-tissue innervation in an infant born at term and lacking genetic defects in the SMN1 and IGHMBP2 genes. The signs characterizing the muscle-tissue immaturity in question are the presence of prima- ry myotubes with prepatterned clusters devoid of nerve terminals, as well as a lack of synapses and the appearance of numerous unmyelinated axons within a single Schwann cell. During human myogenesis, the primary myotubes that are the predominant feature of developing muscles 10 weeks into gestation begin to increase in diameter, by fusing site to site with the myoblast to create more mature myotubes 

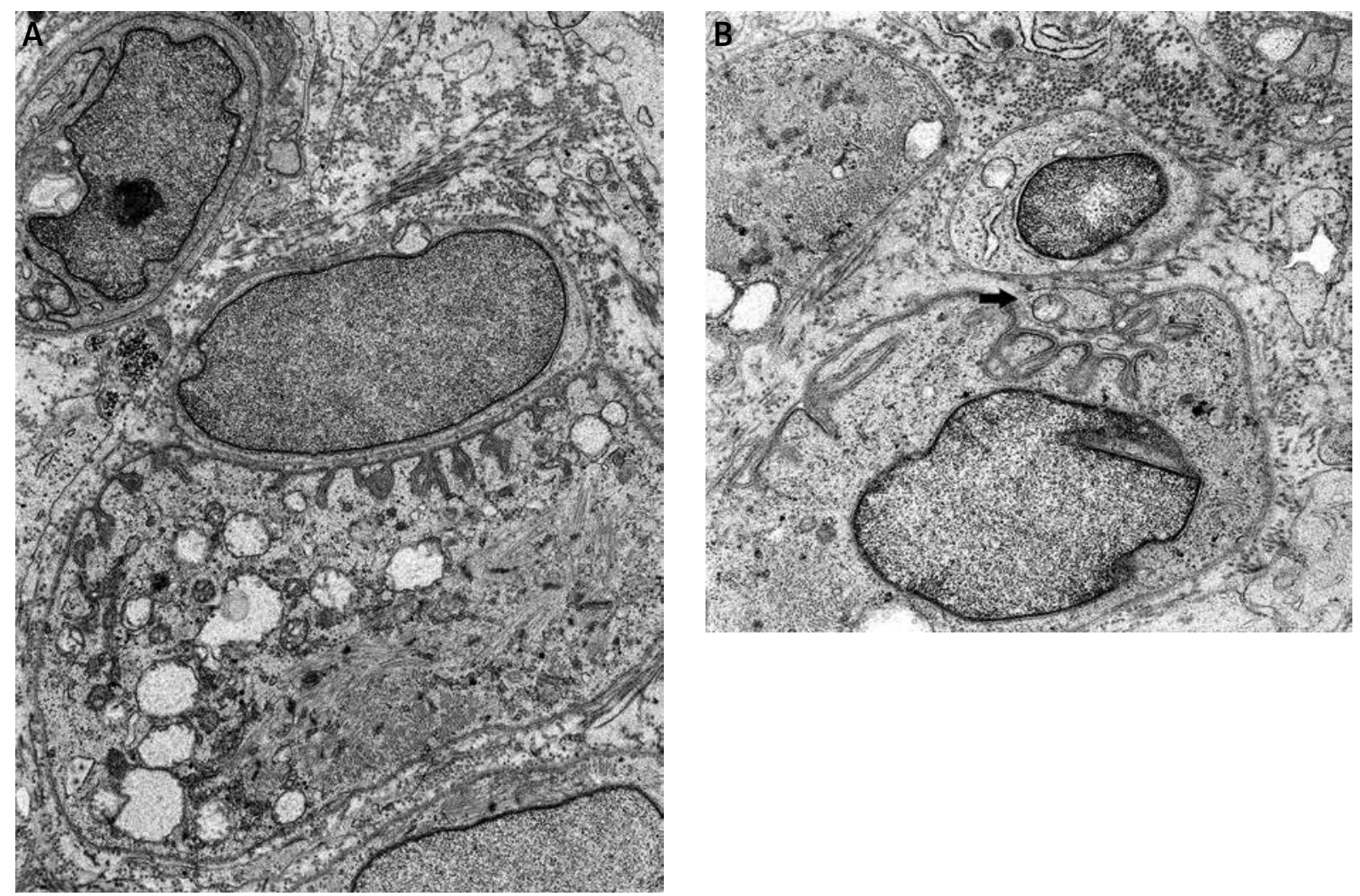

Fig. 3. A, B) Primary myotubes with prepatterned AChR clusters devoid of terminal axons (arrow); $\times 18000$.
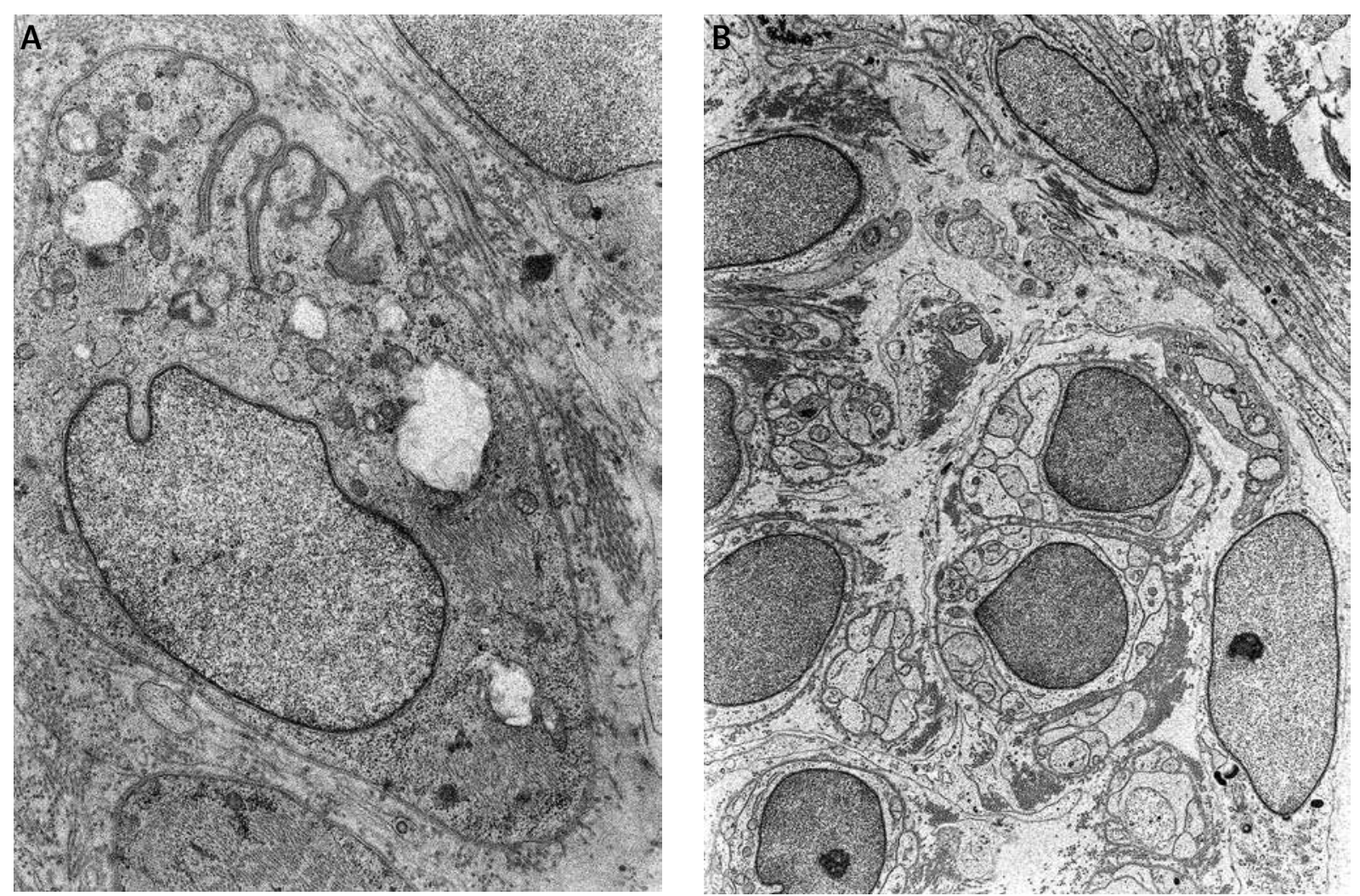

Fig. 4. A) Prepatterned junctional folds penetrating into the myotube interior; $\times 21000$. B) Unmyelinated multiaxonal intramuscular nerves; $\times 9000$. 
$[2,4]$. Mature myotubes, with their characteristic pattern of 2-3 cells at various stages of differentiation closely connected and enveloped by lamina basalis, are normally observed until 16-17 weeks into gestation $[2,4]$. The presence in our infant of muscle cells at the myotube stage offers a clear indication that the process of muscle maturation has been arrested. The presence of multiaxonal unmyelinated intramuscular nerves is a further proof of the maturation failure seen in the investigated muscle. In humans, the process of peripheral nerve myelinisation begins 17 weeks into the gestation period, and terminates shortly before birth $[2,3,5,15]$.

The prepatterned clusters observed in the investigated myotubes may be regarded as the most surprising finding seen never before in human synaptogenesis [5], though found in mutated mice lacking motor nerves [18].

Recent studies have suggested that ectopic MuSK expression is sufficient to establish prepatterned AChR clusters accumulated in the centre of the muscle cell prior to the arrival of motor growth cones [12]. Motor axons will grow through the region where these prepatterned AChR clusters reside, and induce neuromuscular synapses by incorporating the prepatterned cluster into the neuromuscular junction (NMJ) [11]. This formation of the neuromuscular synapse can be divided [10] into: an early phase closely associated with the formation of prepatterned structures and navigation of growth cones, and a late phase during which growth cones make contact with AChR clusters and become NMJs. The prepatterned phenomenon observed by us seems to indicate that, although the myotubes were ready to accept the contact with the terminal axons, the nerves were not able to do so. While molecular analysis eliminated the possibility of mutation in the SMN1 and IGHMBP2 genes, we suggest that a new and as-yet unknown genetic defect is responsible for such great immaturity. In addition, the "naked" prepatterning clusters observed on the surfaces of myotubes with arrested maturation may be the main sign of the innervation failure.

\section{Acknowledgements}

This study was supported by grant N N401 011038 (MJ) from the Ministry of Science and Higher Education of Poland.

\section{References}

1. Burden SJ. The formation of neuromuscular synapses. Genes Dev 1998; 12: 133-148.

2. Fidziańska A. Electron microscopic study of the development of human foetal muscle, motor end-plate and nerve. Preliminary report. Acta Neuropathol 1971; 17: 234-247.

3. Fidziańska A. Fine structure of human fetal nerve. Pol Med Sci Hist Bull 1976; 15: 281-289.

4. Fidziańska A. Human ontogenesis. I. Ultrastructural characteristics of developing human muscle. J Neuropathol Exp Neurol 1980; 39: 476-486.

5. Fidziańska A. Human ontogenesis. II. Development of the human neuromuscular junction. J Neuropathol Exp Neurol 1980; 39: 606-615.

6. Gamble HJ. Further electron microscope studies of human foetal peripheral nerves. J Anat 1966; 100: 487-502.

7. Grohmann K, Schuelke M, Diers A, Hoffmann K, Lucke B, Adams C, Bertini E, Leonhardt-Horti H, Muntoni F, Ouvrier R, Pfeufer A, Rossi R, Van Maldergem L, Wilmshurst JM, Wienker TF, Sendtner M, Rudnik-Schöneborn S, Zerres K, Hübner C. Mutations in the gene encoding immunoglobulin mu-binding protein 2 cause spinal muscular atrophy with respiratory distress type 1 . Nat Genet 2001; 29: 75-77.

8. Hall ZW, Sanes JR. Synaptic structure and development: the neuromuscular junction. Cell 1993; 72: 99-121.

9. Jedrzejowska M, Borkowska J, Zimowski J, Kostera-Pruszczyk A, Milewski M, Jurek M, Sielska D, Kostyk E, Nyka W, Zaremba J, Hausmanowa-Petrusewicz I. Unaffected patients with a homozygous absence of the SMN1 gene. Eur J Hum Genet 2008; 16: 930-934.

10. Jing L, Lefebvre JL, Gordon LR, Granato M. Wnt signals organize synaptic prepattern and axon guidance through the zebrafish unplugged/MuSK receptor. Neuron 2009; 61: 721-733.

11. Jing L, Gordon LR, Shtibin E, Granato M. Temporal and spatial requirements of unplugged/MuSK function during zebrafish neuromuscular development. PLoS One 2010; 5: e8843.

12. Kim N, Burden SJ. MuSK controls where motor axons grow and form synapses. Nat Neurosci 2008; 11: 19-27.

13. Lin W, Burgess RW, Dominguez B, Pfaff SL, Sanes JR, Lee KF. Distinct roles of nerve and muscle in postsynaptic differentiation of the neuromuscular synapse. Nature 2001; 410: 10571064.

14. Mazhar S, Herbst R. The formation of complex acetylcholine receptor clusters requires MUSK kinase activity and structural information from the MuSK extracellular domain. Mol Cell Neurosci 2012; 49: 475-486.

15. Ochoa J. The sural nerve of the human foetus: electron microscope observations and counts of axons. J Anat 1971; 108: 231-245.

16. Panzer JA, Song Y, Balice-Gordon RJ. In vivo imaging of preferential motor axon outgrowth to and synaptogenesis at prepatterned acetylcholine receptor clusters in embryonic zebrafish skeletal muscle. J Neurosci 2006; 26: 934-947.

17. Sanes JR, Lichtman JW. Development of the vertebrate neuromuscular junction. Annu Rev Neurosci 1999; 22: 389-442.

18. Yang X, Arber S, William C, Li L, Tanabe Y, Jessell TM, Birchmeier C, Burden SJ. Patterning of muscle acetylcholine receptor gene expression in the absence of motor innervation. Neuron 2001; 30: 399-410. 